\title{
Can tactile neglect occur at an intra-limb level? Vibrotactile reaction times in patients with right hemisphere damage
}

\author{
J.B. Mattingley and J.L. Bradshaw \\ Department of Psychology, Monash University, Clayton, Victoria 3168, Australia
}

Correspondence to: J.B. Mattingley at above address

\begin{abstract}
Visual reaction time (RT) studies on patients with right hemisphere (RH) damage have demonstrated that the attentional imbalance to stimuli occupying left and right positions exists even within the "intact" ipsilesional hemifield. The purpose of the present study was to test whether such patients might also exhibit relative left-sided impairments in the tactile modality, where stimuli and responses involve the index and middle fingers of the non-hemiplegic ipsilesional hand. Eight patients with RH damage, and eight matched normal controls, were tested using a vibrotactile choice RT paradigm, with the responding hand held in prone or supine posture, and located either at the body midline, or in left or right hemispace. Patients showed significantly slower RTs with the left than the right finger in both hand postures, a difference which remained constant as a function of the hemispatial location of the responding hand. In the prone posture, patients' left finger RTs were slower than those of controls, who showed no difference between left and right finger RTs, while their right finger RTs were faster than those of controls. In the supine posture, both patients and controls exhibited slower left than right finger RTs, though in controls the left finger disadvantage was attributed to biomechanical rather than attentional factors. Patients also made more errors with left than right finger stimuli, both as failures of detection and as incorrect responses, while controls made fewer errors overall and showed no differences between fingers. These data demonstrate a bias in the distribution of attention to tactile stimuli at an intra-limb level, and suggest that the attentional imbalance created by RH damage may be supramodal.
\end{abstract}

Keywords: Allesthesia - Allochiria - Attention - Hemispace - Neglect - Right hemisphere - Vibrotactile

\section{INTRODUCTION}

The disorder of unilateral spatial neglect ("neglect") has been documented in such modalities as the visual (Brain, 1941; Critchley, 1966; see also Jeannerod, 1987; Robertson and Marshall, 1993), auditory (e.g. Altman et al., 1979; Bisiach et al., 1984; De Renzi et al., 1989a), olfactory (Bellas et al., 1988), and tactile (De Renzi et al., 1970; Vallar et al., 1991; Villardita, 1987). From a clinical perspective, the last of these, tactile neglect, may be the most salient manifestation of a lateralized attentional impairment, since a lack of awareness of tactile stimuli on the contralesional side may hinder rehabilitative interventions based on body awareness (e.g. Robertson and Cashman, 1991). On the other hand, directing patients' attention to the affected side may reduce the extent of tactile neglect (Weiskrantz and Zhang, 1987). The purpose of the present study was to examine attentional allocation, in right hemisphere $(\mathrm{RH})$ lesioned patients, to discrete tactile stimuli restricted to the ipsilesional hand.

Most previous studies of tactile neglect have required patients to locate objects in a maze or on a response board, in the absence of vision (De Renzi et al., 1970; Bisiach et al., 1985; Villardita, 1987; Weintraub and Mesulam, 1987; Vallar et al., 1991; Caneman et al., 1992). In such tasks, the presence of tactile neglect is indicated when patients fail, or are slow, to detect targets in the contralesional hemispace, using the ipsilesional upper limb. At least one other study employed a tactile analogue of the standard line bisection test, but failed to demonstrate significant tactile neglect (Fujii et al., 1991).

The common underlying feature of most paradigms used to document tactile neglect is that they involve a substantial exploratory-motor component. Thus, patients are reliant not only upon somatosensory information from the ipsilesional hand, but also on kinesthetic signals from receptors in muscles, tendons and joints, in addition to feedback on kinematic parameters required for adequate motor control. Therefore, these studies are perhaps best categorized as demonstrating "tactile-kinaesthetic" or "exploratory-motor" neglect, rather than "pure" tactile neglect. The terminology suggested here is not 
trivial, since there is now substantial evidence, in patients with RH damage, of deficits in the timing (Heilman et al., 1985a; Mattingley et al., 1992a) and spatial extent (Bisiach et al., 1990; Tegnér and Levander, 1991) of contralesionally directed movements of the ipsilesional limb. Thus, in any task involving active exploration of peripersonal space, the relative contributions of deficits in perceptual (tactile) and response (exploratory-motor) components remain unclear.

In contrast, there is at least one quantitative study which has demonstrated the existence of tactile neglect in the absence of an exploratory-motor response. Pierson-Savage et al. (1988) used a simple reaction time (RT) paradigm to measure the responses of RH lesioned patients to discrete vibrotactile stimuli. Patients had a small vibrotactile transducer attached to the index finger of their ipsilesional hand, which was itself located either in its own (right) or the opposite (left) hemispace. Responses were made by depressing a microswitch situated beneath the responding finger. Eight unrehabilitated patients showed substantially longer RTs with the hand located in the left than the right hemispace, suggesting impaired attention to discrete tactile stimuli in left hemispace. This study provides the most unequivocal evidence of "tactile" (or perhaps "tactuomotor") neglect.

While a deficit in detecting and responding to tactile stimuli in the contralesional hemispace is consistent with models which suggest that neglect is a hemispace phenomenon, in which the body's midsagittal plane acts as a dividing line between the unattended left and attended right halves of space (Heilman et al., 1985b; Mesulam, 1985), it is also consistent with the so-called "gradient" or "vector" model (Kinsbourne, 1987, 1993; see also Mattingley et al., 1992b for review). The latter is based in part on the observation that $\mathrm{RH}$ damaged patients may respond more slowly to a visual stimulus occupying a relative left position within the ipsilesional visual field, than to a stimulus occupying a relative right position in the same field (Làdavas et al., 1990).

Thus, there is clearly an attentional deficit even within the "intact" hemispace. Moreover, since the RTs of such patients to relative right-sided visual stimuli are similar to, and often faster than, those of controls, it has been suggested that patients direct their attention preferentially (and perhaps automatically) towards the rightmost visual stimulus in an array (De Renzi et al., 1989b; D'Erme et al., 1992), even after recovery from clinical signs of neglect (Mattingley et al., 1994). The model proposed by Kinsbourne $(1987,1993)$ neatly encompasses these findings, by assuming that each hemisphere controls a contralesionally directed attentional vector. Although the vectors are usually well balanced, damage to the $\mathrm{RH}$ releases the left hemisphere (rightward) vector from inhibition, thereby producing an attentional imbalance or "gradient". Since spatial attention can be allocated widely or diffusely, or alternatively as a narrow beam or spotlight, the same gradient in processing resources may exist on different spatial scales, so that there may be neglect of whole objects on the left side of a horizontal array, or neglect of the left sides of several objects within the array (Gainotti et al., 1972).

The purpose of the present study was to test whether RH damaged patients might also exhibit relative left-sided impairments in the tactile modality. Such a study has not been undertaken previously, ${ }^{1}$ though data of this kind are potentially important for determining whether spatial attention is subserved by similar mechanisms across different sensory modalities. More specifically, since RT differences exist between relative left and right visual stimuli within the ipsilesional field, do such differences also exist between RTs to relative left and right tactile stimuli?

Whereas in vision, defining the positions of stimuli along the horizontal axis, even within a restricted region of space, is relatively straightforward, the assignment of left and right in the tactile modality is somewhat more difficult, especially since patients typically have left-sided sensory and motor impairments which preclude the recording of tactile RTs from the contralesional limb. We devised a vibrotactile RT paradigm similar to that used by Pierson-Savage et al. (1988), except that patients received stimuli from one of two transducers, attached to the index and middle fingers of the intact ipsilesional (right) hand. In consequence, unlike the study of Pierson-Savage

\footnotetext{
${ }^{1}$ Since submission of this manuscript, Moscovitch and Behrmann (1994) have shown in an elegant study that unilateral neglect patients may exhibit contralesional extinction with double simultaneous presentation of tactile stimuli on the medial and lateral surfaces of the ipsilesional wrist, irrespective of hand posture (prone or supine). However, their study did not address the issue of patients' responses to single (as opposed to double simultaneous) lateralized stimuli, nor did it examine the possible influence of hemispatial location of the ipsilesional arm upon patient performance. Moreover, their method of stimulus presentation (manual delivery using cotton wool or light finger touch) was not standardized with respect to duration, intensity, etc., in contrast to the technique adopted in our study where such parameters were precalibrated and computer controlled. Finally, Moscovitch and Behrmann (1994) did not present data from matched, healthy subjects with which to compare the performances of their patient group.
} 
et al. (1988), the present experiment involved the measurement of choice, rather than simple RTs.

Note that the vibrotactile RT task, like the paradigms reviewed above and used to document tactile neglect, is reliant upon kinesthetic signals from receptors in muscles, tendons and joints, which provide information on the spatial location of the responding hand with respect to the midline. However, in contrast to previously adopted paradigms the vibrotactile RT task does not require exploratory-motor limb movements. There is substantial evidence from studies of normal healthy subjects indicating that patterns of performance on the vibrotactile RT task are determined by the relative spatial locations of the relevant limbs. For example, Bradshaw et al. (1983) showed that in a simple vibrotactile RT task either hand was faster when it lay to the right of the midline, an effect which was lost with $90^{\circ}$ head turns to unconfound head and body coordinates. Under such conditions performance was superior when the hand lay in right hemispace with respect to the head rather than the body, indicating that hemispace effects depend more on head than body coordinates. Similarly, PiersonSavage and Bradshaw (1987) showed that, again in a simple vibrotactile RT task, with either hand tested at $90^{\circ}, 45^{\circ}$ or $15^{\circ}$ to left or right of the midline, or at the midline itself, performance slowed with either hand somewhere beyond $45^{\circ}$ to left of the midline.

Further indication of the role of spatially directed attention in such tasks comes from the observation of Pierson et al. (1991), this time in a bimanual choice RT paradigm; performance was slower when the two arms each crossed the body midline, but under such circumstances there was significant response facilitation when the subject could look at (compared with away from) the responding hand (see also Bradshaw et al., 1993). These performance asymmetries, whether or not modulated by overt directed attention (gaze), clearly involve relative differences in the strength of deployment of covert attentional processes with respect to major coordinate systems.

In the present study, the two fingers of the ipsilesional hand occupied relative left and right positions in space. Patients responded to each vibrotactile stimulus by pressing the appropriate member of a pair of horizontally adjacent microswitches. Reaction times were measured with the responding hand in either a prone (i.e. with the palm facing down) or supine (i.e. with the palm facing up) posture, to control for any mechanical differences between the two fingers. The responding hand was located either opposite the body midline, or in either hemispace. If attention is indeed subserved by contralaterally op- posed vectors, as suggested by Kinsbourne (1987, 1993), then RH damaged patients should exhibit slower RTs to stimuli delivered to the finger occupying the left compared with the right spatial position, regardless of hand posture or the location of the responding limb in relation to the body midline. Alternatively, if neglect reflects an attentional deficit restricted to the left hemispace (Heilman et al., 1985b; Mesulam, 1985), then the location of the responding hand relative to the body midline (but not the positions of the two fingers) should determine the pattern of RTs.

\section{METHODS}

\section{Subjects}

Eight patients with unilateral $\mathrm{RH}$ damage and eight sex- and age-matched healthy controls participated. Age, sex and clinical details for the patient group are provided in Table I. All patients had suffered strokes in the territory of the middle cerebral artery, except Patients 3 and 8, whose lesions indicated posterior cerebral artery involvement. Lesion location was inferred from clinical examination and confirmed by cranial computed tomography (CT) scan. Patients were screened for gaze disturbances, and visual fields were examined by confrontation testing. The mean (S.D.) age of the patient group was 62.6 (11.8) years, while that of controls was $66.5(8.2)$ years, $F(1,14)=$ 0.586 , N.S. All subjects were assessed as being righthanded from their performance on a 10 item questionnaire (Patterson and Bradshaw, 1975). Patients exhibited normal sensory and motor function in their preferred (ipsilesional) upper limbs.

\section{Testing for unilateral neglect}

Patients completed a standard clinical protocol prior to participating in the main experimental investigation. Each patient was given a line cancellation test (Albert, 1973), a circle cancellation test (Ellis et al., 1987), and the Star Cancellation task from the Behavioural Inattention Test (Wilson et al., 1987). Patients were also given a line bisection test, consisting of 10 horizontal lines varying in length from 80 to $170 \mathrm{~mm}$ in $10 \mathrm{~mm}$ increments. These lines were centred on a single sheet of A4 paper in pseudorandom order, and drawn through a white cardboard mask with a central window which exposed one line at a time. Deviation from the true midpoint of each line was measured to the nearest millimetre.

Each test was placed directly in front of the patient and centred at the body midline. Patients used their preferred (ipsilesional) hands to hold the pencil. The 
TABLE I. Patient clinical details and performances on cancellation (percentage omissions) and line bisection (mean error in $\mathrm{mm}$ ) tests

\begin{tabular}{|c|c|c|c|c|c|c|c|c|c|}
\hline \multirow[b]{2}{*}{ Patient } & \multirow[b]{2}{*}{ Age/Sex } & \multirow[b]{2}{*}{ Lesion } & \multirow[b]{2}{*}{ Aetiology } & \multirow[b]{2}{*}{ Post-stroke } & \multirow[b]{2}{*}{ VFA } & \multicolumn{4}{|c|}{ Clinical tests } \\
\hline & & & & & & $A L$ & $\mathrm{CC}$ & SC & LB \\
\hline 1 & $56 / M$ & $T$ & Ischaemia & 2 & NAD & 0 & 0 & 4 & 1 \\
\hline 2 & $67 / F$ & PS & Haemorrhage & 5 & LHH & 0 & 30 & 67 & 5 \\
\hline 3 & $79 / M$ & OS $^{1}$ & Ischaemia & 13 & $\mathrm{LHH}$ & 33 & 10 & 52 & 34 \\
\hline 4 & $41 / \mathrm{M}$ & $s$ & Haemorrhage & 1 & $\mathrm{LHH}$ & 0 & 0 & 20 & 6 \\
\hline 5 & $60 / \mathrm{M}$ & OP & Haemorrhage & 1 & $\mathrm{LHH}$ & 30 & 25 & 56 & 27 \\
\hline 6 & $66 / M$ & FP & Haemorrhage & 48 & NAD & 5 & 20 & 22 & 2 \\
\hline 7 & $58 / \mathrm{M}$ & FP & Ischaemia & 2 & NC & 0 & 0 & 0 & 0 \\
\hline 8 & $74 / F$ & OT & Ischaemia & 14 & $\mathrm{LHH}$ & 23 & 0 & 24 & 32 \\
\hline
\end{tabular}

Post-stroke = time of testing (weeks); VFA, visual field assessment; $F$, frontal; O, occipital; $P$, parietal; T, temporal; S, subcortical; LHH, left homonymous hemianopia; NAD, no abnormalities detected; NC, not conducted; AL, Albert lines; CC, circle cancellation; SC, Star cancellation; LB, line bisection (error in $\mathrm{mm}$ ).

1 Patient also had small cerebellar infarcts bilaterally.

percentage of omissions on each of the cancellation tests, and mean bisection error (in $\mathrm{mm}$ ), are shown in Table I. Positive numbers for line bisection indicate a mean rightward error. Patients were selected to be relatively heterogeneous in terms of their performance on standard clinical tests, so that we could examine the extent to which the magnitude of any RT differences may have been correlated with deficits on clinical measures.

\section{Apparatus}

A detailed description of the stimulus delivery system has been provided elsewhere (Wood et al., 1988). Briefly, two Oticon-A vibrotactile transducers (47 $\Omega$ impedance, $17 \mathrm{~mm}$ active surface) were used to deliver stimuli. These were driven by oscillators under the control of a Toshiba 3100e laptop computer. Vibrotactile stimuli $(250 \mathrm{~Hz})$ were delivered at an intensity of $4.0 \mathrm{~V}$ peak-to-peak for $80 \mathrm{~ms}$, with a ramp time of $20 \mathrm{~ms}$, producing a clearly perceptible signal. Two response buttons $(10 \mathrm{~mm}$ diameter microswitches) were mounted side by side on a wooden box with a horizontal separation of $40 \mathrm{~mm}$. The response box was itself attached, via two laterally positioned adjustable arms (length $=125 \mathrm{~mm}$ ), to a wooden base. Earphones were worn by subjects to eliminate possible auditory cues arising from the transducers.

\section{Procedure}

Subjects sat at a table with their right hands located on the response board. Vibrotactile transducers were attached to the upper surface of the index and middle fingers, above the second phalanx. This left the finger pads free to rest upon each of the response buttons. For trials in which the hand was held in a prone posture, the response box sat on the wooden base, while for trials in which the hand was held in a supine posture, the box was raised from the base by means of the adjustable arms. In both postures, subjects' palms rested either above (prone) or below (supine) the box, with the index and middle fingers curled around so that the response buttons were activated by pressure toward the body. The centre of the response box was located either directly opposite the body midline, or $250 \mathrm{~mm}$ into left or right hemispace.

Subjects were required to fixate directly ahead throughout the experiment, and were instructed to respond as fast as possible by pressing the button beneath the finger receiving a stimulus. All aspects of stimulus control and response recording were controlled. by the computer. Stimuli were delivered in blocks of 10 trials. An equal number of stimuli in each block was delivered in a pseudorandom order to the two fingers. There were 12 blocks of trials, two for each posture (prone, supine) at each of three different spatial locations (left, midline, right), making a total of 12 blocks (120 trials) per subject. The response board position and hand posture were counterbalanced both within and between subjects. Vibrotactile transducers and response buttons were interchanged halfway through the experimental session. Each subject was given at least one block of practice trials for each hand posture, with the response board located at the midline. Some patients required additional practice trials, which were allowed as necessary.

In addition to recording reaction times, the computer also recorded incorrect responses for each condition. Mean RTs for each condition were based only upon correct responses. Reaction times below $150 \mathrm{~ms}$

70 Behavioural Neurology . Vol 7 . 1994 
TABLE II. Patients' mean reaction times and combined errors for left (LF) and right (RF) fingers in prone and supine hand postures

\begin{tabular}{|c|c|c|c|c|c|c|}
\hline \multirow[b]{3}{*}{ Patient } & \multicolumn{4}{|c|}{ Reaction times (ms) } & \multirow{2}{*}{\multicolumn{2}{|c|}{$\begin{array}{c}\text { Errors }(/ 120) \\
\text { Prone + Supine }\end{array}$}} \\
\hline & \multicolumn{2}{|c|}{ Prone } & \multicolumn{2}{|c|}{ Supine } & & \\
\hline & LF & RF & LF & RF & LF & RF \\
\hline 1 & 546 & 436 & 427 & 385 & 7 & 8 \\
\hline 2 & 607 & 520 & 663 & 557 & 15 & 5 \\
\hline 3 & 288 & 246 & 270 & 265 & 10 & 6 \\
\hline 4 & 428 & 383 & 454 & 386 & 8 & 4 \\
\hline 5 & 412 & 303 & 337 & 323 & 12 & 3 \\
\hline 6 & 416 & 431 & 472 & 462 & 20 & 7 \\
\hline 7 & 465 & 441 & 561 & 508 & 3 & 8 \\
\hline 8 & 484 & 475 & 501 & 470 & 5 & 3 \\
\hline
\end{tabular}

were discarded as they were considered to be anticipations, while those above $2000 \mathrm{~ms}$ were discarded as omissions. Reaction times that were more than 3 standard deviations from the subject's overall mean were replaced with that cutoff value. Discarded and altered trials were not repeated. In the event, such instances were rare, and all subjects (patients and controls) comfortably exceeded the predetermined criterion of $80 \%$ correct.

\section{RESULTS}

\section{Reaction times}

Mean RTs were analysed in a mixed model analysis of variance (ANOVA) with Group (patients, controls) as a between-subjects factor and Hand Posture (prone, supine), response Board Location (left, midline, right), and Finger (left, right) as within-subjects factors. There was a significant main effect of Finger $[F(1,14)=45.739, p<0.001]$, and significant threeway interactions of Group by Hand Posture by Board Location $[\mathrm{F}(2,28)=4.789, p<0.05]$, and Group by Hand Posture by Finger $[\mathrm{F}(1,14)=5.091, p<0.05]$. These interactions were examined further in separate ANOVAs for each subject group, using the same within-subjects factors as in the main analysis.

For patients, there was a significant simple main effect of Finger $[F(1,7)=16.336, p<0.01]$, with RTs for the left finger $(458 \mathrm{~ms}$ ) being $46 \mathrm{~ms}$ slower than RTs for the right finger ( $412 \mathrm{~ms})$. There were no significant simple main effects of Hand Posture $[\mathrm{F}(1,7)=0.305$, N.S. $]$ or Board Location $[\mathrm{F}(2,14)=$ $1.166, p>0.05]$, and no significant interactions, indicating that patients' RTs were only affected by the relative positions (left or right) of the two fingers, and not by the posture or hemispatial position of the operating hand. Thus, left finger disadvantages for left, midline and right board locations were 45, 55 and $39 \mathrm{~ms}$, respectively. Table II shows mean left and right finger RTs for individual patients, collapsed across Board Location, as a function of Hand Posture.

For healthy controls there was also a significant simple main effect of Finger $[F(1,7)=37.453$, $p<0.001$ ], and a significant two-way interaction of Hand Posture by Board Location $[\mathrm{F}(2,14)=6.626$, $p<0.01]$. Since the interaction did not involve the variable of interest (Finger), it will not be considered further here. There were, however, two strong interactive trends involving Finger position. The first of these, Hand Posture by Finger $[\mathrm{F}(1,7)=4.836, p<$ 0.07 , indicated that while RTs in the supine posture were slower for the left finger $(496 \mathrm{~ms})$ than the right $(404 \mathrm{~ms})$, there was essentially no difference between RTs for left $(438 \mathrm{~ms})$ and right $(437 \mathrm{~ms})$ fingers with the hand in prone posture. The second trend, Board Location by Finger $[\mathrm{F}(2,14)=3.512, p<0.06]$, indicates that although right finger RTs were faster than left finger RTs overall, the magnitude of the discrepancy changed with the location of the responding hand (left: $48 \mathrm{~ms}$; midline: $72 \mathrm{~ms}$; right: $22 \mathrm{~ms}$ ). Thus, in contrast with patients, a difference between left and right finger RTs in healthy controls occurred only with a supine hand posture, and was more pronounced with the operating hand located at the midline or in contralateral hemispace, than in ipsilateral hemispace.

Figure 1 shows the mean RTs of left and right fingers for each subject group, separately for the two hand postures. With the hand held in prone posture, patients showed a strong effect of finger position, while controls showed no effect. It is also important to note that patients' left finger RTs were slower than those of controls, while their right finger RTs were actually faster than those of controls. In contrast, the pattern of RTs with a supine posture was similar in the two groups, although left finger RTs were faster in patients than controls, while right finger RTs were slower

\section{Errors}

Although subjects made relatively few errors, these were also examined in order to determine whether they occurred with a different frequency with left and right fingers. Given their difficulties in detecting and responding to left-sided stimuli, it was predicted that patients would exhibit a higher number of left finger errors, while controls would show a roughly equal number of left and right finger errors. To obtain more reliable means, errors for each group were 

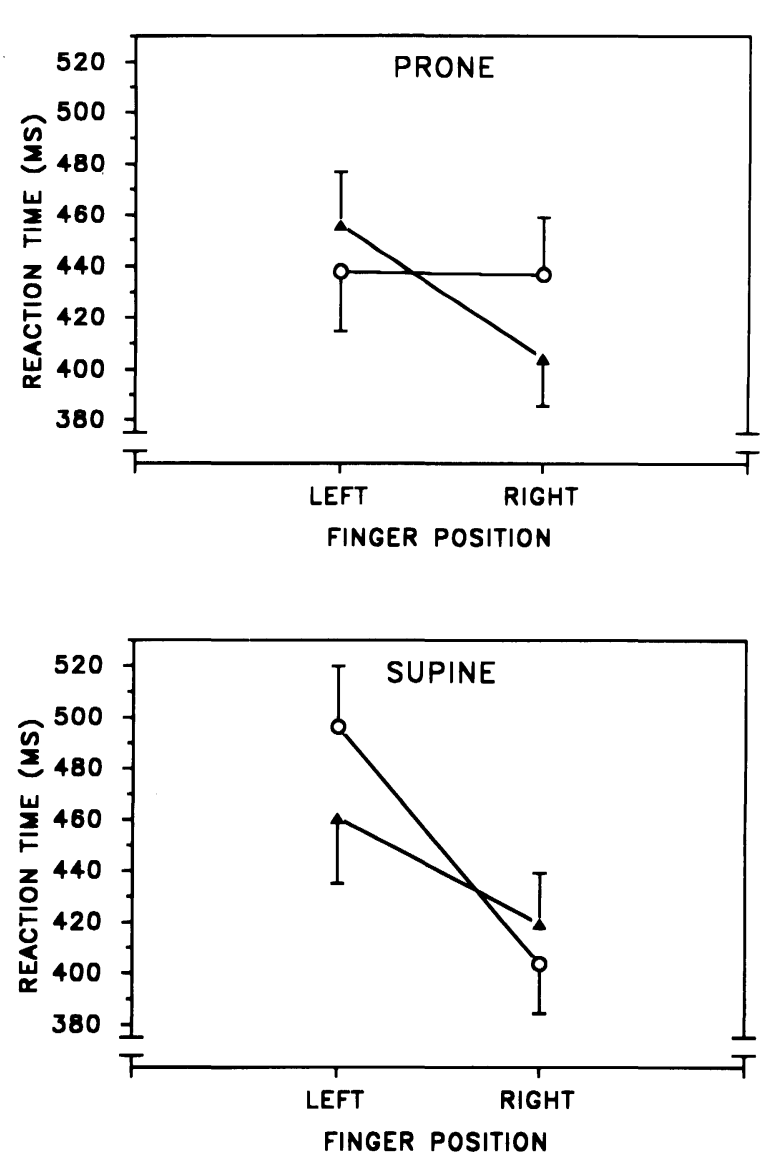

FIG. 1. Mean choice reaction time ( \pm 1 S.E.) as a function of relative finger position, plotted separately for prone (above) and supine (below) postures. Open circles: controls; filled triangles: $\mathrm{RH}$ damaged patients.

collapsed across Hand Posture and Board Location. In the present paradigm, errors could be of two types; missed stimuli, in which the subject failed to respond within $2000 \mathrm{~ms}$, and incorrect responses, in which the incorrect button was pressed, e.g. a right button press to a left-sided stimulus.

Combined error data (missed stimuli and incorrect responses) from individual patients are shown in Table II, collapsed across the factors of Board Location and Hand Posture. Analysis of these combined error data, with an a priori prediction of greater leftthan right-sided errors, revealed significantly more left than right finger errors in patients $[t(7)=2.130$, $p<0.05$, one-tailed], but no significant difference between fingers in controls $[t(7)=1.069, p>0.05$, onetailed]. Figure 2 shows the means for each error type for the two subject groups, as a function of finger position. Overall, patients missed more stimuli and made more incorrect responses than controls. Of particular interest is the observation that patients
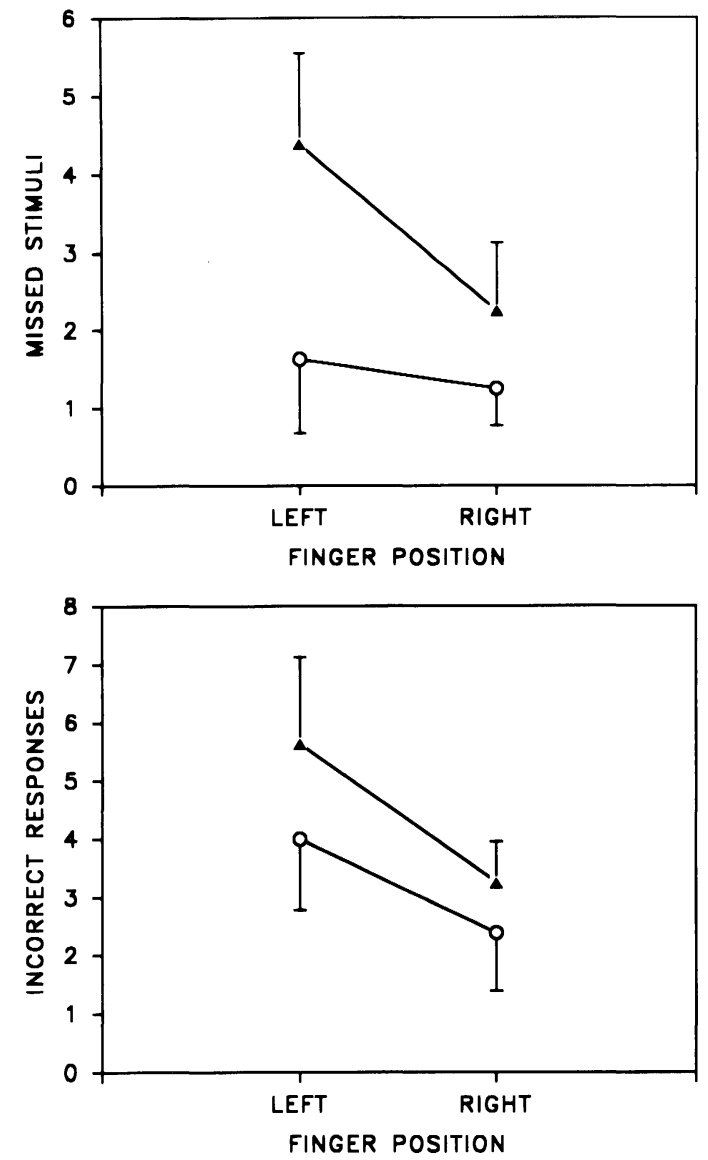

Fig. 2. Mean number of errors from 120 trials $( \pm 1$. S.E.) as a function of relative finger position, plotted separately for missed stimuli (above) and incorrect responses (below). Open circles: controls; filled triangles: $\mathrm{RH}$ damaged patients.

missed substantially more left finger stimuli than right finger stimuli, while controls showed little difference between fingers. Both patients and controls made more incorrect responses to left than right finger stimuli, though the gradient for patients was marginally steeper.

\section{Relation between vibrotactile and clinical test performance}

It has been established that there are strong correlations between several clinical tests of unilateral neglect (Halligan et al., 1989; Kinsella et al., 1993). These tests share at least one common feature, namely, the requirement of adequate visual attention for successful performance. It would therefore be of interest to determine whether the extent of impairment documented by such tests might be associated with errors in detecting or responding to left-sided tactile stimuli, or with prolonged left finger RTs. To this end, we conducted correlational analyses to com- 
TABLE III. Pearson product-moment correlation coefficients for cancellation, line bisection, left/right finger reaction time differences, and left/right finger error differences

\begin{tabular}{|c|c|c|c|c|c|c|c|c|}
\hline Task & 1 & 2 & 3 & 4 & 5 & 6 & 7 & 8 \\
\hline 1. L-R finger RT (prone) & - & & & & & & & \\
\hline 2. L-R finger RT (supine) & 0.28 & - & & & & & & \\
\hline 3. L-R finger errors (incorrect) & -0.23 & -0.53 & - & & & & & \\
\hline 4. L-R finger errors (misses) & 0.13 & 0.29 & 0.17 & - & & & & \\
\hline 5. Line bisection (mean) & -0.61 & -0.04 & 0.30 & -0.32 & - & & & \\
\hline 6. Albert lines & -0.57 & -0.02 & 0.38 & -0.12 & $0.96^{\star \star}$ & - & & \\
\hline 7. Circle cancellation & 0.51 & -0.05 & -0.08 & 0.05 & 0.05 & 0.17 & - & \\
\hline 8. Star cancellation & 0.08 & -0.14 & 0.04 & -0.12 & 0.50 & 0.50 & $0.80^{*}$ & - \\
\hline
\end{tabular}

* Significant at $\alpha=0.05$

** Significant at $\alpha=0.01$.

pare error and RT differences between left and right fingers, with cancellation test omissions and line bisection errors. The resulting correlation matrix is shown in Table III.

Of course, such an analysis is likely to result in a high Type II error due to the relatively small number of patients. However, even with $\alpha=0.10$, there were no significant correlations between errors or RTs on the vibrotactile task, and performances on any of the visually based clinical tests. The largest positive correlation was between prone posture RT differences and performance on the circle cancellation test $(r=0.51)$, though there were also non-significant negative correlations between the same RT differences, and the Albert lines $(r=-0.57)$ and line bisection tests $(r=-0.61)$. In contrast, significant correlations were found between performances on the Albert lines and line bisection tests $(r=0.96)$, and between performances on the circle cancellation and star cancellation tests $(r=0.80)$. Our tentative conclusion, therefore, is that the extent of attentional impairment measured in the vibrotactile RT task is not (clearly) related to the degree of visual inattention as indexed by standard clinical tests.

\section{DISCUSSION}

The results provide evidence in support of Kinsbourne's $(1987,1993)$ vector model of spatial attention, which predicts that damage to the $\mathrm{RH}$ releases a strong rightward orienting bias, thereby producing an attentional imbalance across the horizontal dimension of space. Our RH damaged patients were significantly slower to react to vibrotactile stimuli on the left finger than the right finger, with the responding hand held in both prone and supine postures. The hemispatial position of the responding hand, moreover, did not influence patients' ability to allocate attention to vibrotactile stimuli. Thus, we have strong evidence that in the tactile modality, as in vision, "left" and "right" are determined by task demands. It was the relative positions of the stimulated and responding fingers, rather than the absolute location of the ipsilesional hand in peripersonal space, which was critical in modulating patients' attention to tactile stimuli.

In terms of Kinsbourne's vectorial model, the spatial extent of the attentional "gradient" induced by unilateral cerebral damage may be widened or narrowed as a function of specific task demands (Kinsbourne, 1993). Our choice RT task required attention to be narrowly focused upon adjacent fingers of the ipsilesional hand; under such conditions, the hemispatial location of the responding hand was rendered irrelevant. These findings are inconsistent with models which assume that neglect is essentially a hemispace phenomenon (Heilman et al., 1985b, 1987; Mesulam, 1985), though neglect of left hemispace may arise under circumstances in which the task requires spatial attention simultaneously to be distributed on both sides of the midline, as in a bimanual choice RT paradigm. Unfortunately, this possibility is difficult to test in patients with neglect because of primary sensory and motor deficits in the contralesional limbs.

Of course, this is not to suggest that hemispace per $s e$ is unimportant in modulating spatial attention. There is ample evidence that the position of stimuli relative to the body midline plays a key role in determining the allocation of spatial attention in both normals and patients with unilateral lesions (Bradshaw et al., 1987; Heilman et al., 1987; Karnath et al., 1991). More specifically, it has been shown in vibrotactile studies with normals that hemispace effects are present only with simple RT, i.e. where there is no uncertainty as to the location of stimulus and response (Bradshaw et al., 1987). In contrast, these effects are replaced by hand asymmetries with choice RT involving both left and right hands (Bradshaw et al., 1987). The results of Pierson-Savage et 
al. (1988) are in accordance with these findings. In patients with RH damage and left neglect, they found prolonged simple RTs with the ipsilesional hand located in the affected (left) hemispace. One conclusion to be drawn from this finding is that patients may experience difficulties in holding attention within the contralesional hemispace, perhaps as a consequence of (extraneous) competing stimuli on the ipsilesional side, in addition to the well-documented deficit in disengaging and shifting attention contralesionally (Posner et al., 1984, 1987).

To return to the present findings, it is also noteworthy that although the two hand postures resulted in an almost identical pattern of left and right finger RTs for RH damaged patients, the same was not true for controls. Considering first the prone posture, controls showed no difference in their RTs to left and right finger stimuli, suggesting that their attention was evenly divided between the two locations. Patients, however, showed slower RTs to left finger stimuli compared with controls, but were actually faster (by $25 \mathrm{~ms}$ ) than controls to respond to right finger stimuli. This latter finding is in accord with previous visual choice RT studies, which have suggested that left neglect patients tend to focus their attention on the rightmost target location within the right visual field, and therefore respond faster to stimuli at this location than to those occurring on the left (Làdavas et al., 1990). Indeed, it may even be that under circumstances in which there are competing demands from left and right spatial locations, such patients allocate most, if not all, of their attentional resources to the right side, as if the task were one of simple RT.

As a corollary, slower RTs to stimuli on the relative left side in RH damaged patients is therefore likely to be attributable to the cost associated with disengaging focal attention from its ipsilesional location in order to shift it contralesionally (Posner et al., 1984, 1987). In extreme cases, patients fail to detect or respond to contralesional stimuli altogether, a suggestion supported in our study by the significantly higher error rate for the relative left than right finger position. When the attentional imbalance is somewhat less severe, patients may simply be slow to disengage attention from the ipsilesional finger, or to prepare and initiate the appropriate response.

One apparently anomalous result was the pattern of RTs obtained from controls, with the responding hand in a supine posture. Their left finger RTs were substantially slower than those of the right finger, in contrast to the almost identical left and right finger RTs obtained with a prone posture. In fact, this finding is not altogether surprising, since these sub- jects found the supine posture particularly unnatural, especially when the responding hand was located at the body midline or in left hemispace, where normally it operates only infrequently, and rarely (if ever) in the supine (grasping) posture (Bradshaw and Rogers, 1993). Under such conditions, the less skilled middle finger would be particularly disadvantaged. Moreover, some controls admitted to adopting a strategy which involved selectively attending to the index finger in order to compensate for the apparent awkwardness associated with middle finger responses. As a consequence, although left (middle) finger RTs were slower than those of patients, right (index) fingers were slightly faster.

In contrast, patients adjusted well to having their ipsilesional hand in a supine posture, and located beyond its usual operating space. This finding is not in fact counterintuitive, if one considers that due to left hemiplegia, these individuals were now completely reliant upon their ipsilesional hand to operate in both sides of space. In lieu of a hemiplegic left hand, the preferred right hand is now required to grasp and hold objects, a skill which typically requires supination, in both right and left hemispace. Indeed, many rehabilitative interventions require patients to practice such manual skills with the ipsilesional hand located in unfamiliar and awkward postures, on both sides of the body midline. One consequence of this may have been to reduce the apparent cost in left finger RTs with a supine posture, which was evident in normal controls.

This suggestion generates a testable prediction, namely, that those patients with a greater interval post-stroke (i.e. those having more familiarity with awkward ipsilesional hand postures) should show a smaller discrepancy between left and right finger RTs in the supine posture, than those with a relatively short interval poststroke. Our patient data support this prediction. Those individuals tested within 2 weeks post-stroke (Patients 1, 4, 5 and 7 in Table I) showed a mean slowing in left relative to right finger RTs of $53 \mathrm{~ms}$, while those tested at an interval of 1 month or greater (Patients 2, 3, 6 and 8) showed a discrepancy of only $29 \mathrm{~ms}$. This is unlikely to be a consequence of "general" improvements in RT with recovery, since patients tested 1 or 2 weeks poststroke had a similar overall RT (446 ms) to those with a greater interval post-stroke (434 ms).

Although most of the RH damaged patients examined in this study also showed unilateral neglect on visually based clinical tests, there was no significant correlation between the magnitude of impairment on these tests, and left-right finger RT differences in the vibrotactile task. Thus, while our data do not neces- 
sarily provide evidence of modality-specific attentional impairments (cf. Barbieri and De Renzi, 1989; Fujii et al., 1991), they nevertheless point to the existence of one or more separate (as yet unspecified) factors influencing performance on such visual and tactile tasks. One possibility is that clinical cancellation tests emphasize attentional scanning of spatially dispersed stimulus arrays (Kinsella et al., 1993), in addition to complex exploratory-motor limb movements, whereas the vibrotactile task involves detection of spatially discrete stimuli via simple finger movements. It is likely that specific task demands play a critical role in determining the manifestations of unilateral neglect (Mattingley et al., 1994).

Turning finally to the error data, patients made significantly more errors with left than right finger stimuli, either by not responding or by responding incorrectly. In contrast, normal controls showed fewer errors overall, and these were approximately equally distributed between the two fingers. In terms of missed stimuli, it is not clear whether patients failed to respond to more tactile stimuli on the left than the right finger because they failed to detect the stimulus, or because they failed to initiate a response. Indeed, we face the same dilemma in interpreting the RT data, since slow responses can arise from either sensory or motor factors (Bradshaw et al., 1983). One way to resolve this issue would be to ask patients to respond with the opposite finger to that receiving a stimulus, thereby dissociating the two components. However, it is our experience that severely impaired patients often have great difficulty with incompatible stimulus-response tasks.

We also examined incorrect responses, in which subjects responded with the finger not receiving a stimulus. Patients made more incorrect responses with left-sided stimuli (by responding with the right finger), than with stimuli to the right finger. These findings are particularly interesting, because they imply that patients mislocalized sensory impressions to a different position on the same limb. It is difficult to settle upon a precise term for such a disturbance. Meador et al. (1991) have highlighted the confusion surrounding the terms "allochiria", which properly denotes mislocalizations to the opposite side of the body (or space), and "allesthesia", which denotes sensory mislocalization to a remote position on the same limb. Strict interpretation of these definitions suggests that our patients exhibited tactile allesthesia, since their errors arose from an adjacent finger of the ipsilesional hand. However, this term clearly fails to capture the distinct asymmetry of their impairment, a fact which is acknowledged explicitly by the term allochiria.
Rather than proposing yet another term for the deficit exhibited by our patients, we instead suggest that the term allochiria might usefully be extended to include consistent transpositions of stimuli from a contralesional to an ipsilesional location on a single limb. The fact that patients may refer vibrotactile stimuli to a more ipsilesional location, in this case from the left to the right finger of the intact hand, parallels findings in the visual modality, where patients copy elements from the "neglected" to the "intact" side of a figure (Halligan et al., 1992). Of course, our findings in the tactile modality must be viewed with a degree of caution, since the data are limited and because controls exhibited a similar, if smaller, tendency.

To conclude, we have demonstrated that the attentional imbalance accompanying $\mathrm{RH}$ damage operates in the tactile modality, and that it occurs at an intralimb level, where attention to horizontally competing stimuli is biased towards the finger occupying a relative ipsilesional position. With attention narrowly focused in this manner, the absolute location of the responding hand does not affect RTs. This finding is incompatible with models which assume that neglect is restricted to the contralesional hemispace. On the other hand, it closely parallels results of RT studies in the visual modality (e.g. Làdavas et al., 1990), and provides additional support for Kinsbourne's vector model of spatial attention, according to which "the relative spatial position of a stimulus is a major determinant of its standing on the attentional hierarchy, (but) its absolute location is not" (Kinsbourne, 1993, p. 65). Our findings are also consistent with the suggestion (Farah et al., 1989) that at least some of the mechanisms underlying spatial attention are similar, if not identical, across different sensory modalities.

\section{Acknowledgements}

We gratefully acknowledge the assistance of administration and staff at the following institutions: Alfred Hospital, Austin Hospital, Hampton Rehabilitation Hospital, Heidelberg Repatriation Hospital, Kingston Centre, Monash Medical Centre and Royal Talbot Rehabilitation Hospital. We sincerely thank Mike Durham for writing the software, and Rosemary Williams for her assistance in producing the figures. This study was supported by a grant from the Australian Research Council.

\section{REFERENCES}

Albert ML (1973) A simple test of visual neglect. Neurology, 23, 658-664.

Altman JA, Balonov LJ and Deglin VL (1979) Effects of unilateral disorder of the brain hemisphere function in man on directional hearing. Neuropsychologia, 17, 295301. 
Barbieri C and De Renzi E (1989) Patterns of neglect dissociation. Behavioural Neurology, 2, 13-24.

Bellas DN, Novelly RA, Eskenazi B and Wasserstein J (1988) Unilateral displacement in the olfactory sense: a manifestation of the unilateral neglect syndrome. Cortex, 24, 267-275.

Bisiach E, Cornacchia L, Sterzi R and Valler G (1984) Disorders of perceived auditory lateralization after lesions of the right hemisphere. Brain, 107, 37-52.

Bisiach E, Capitani E and Porta E (1985) Two basic properties of space representation in the brain: evidence from unilateral neglect. Journal of Neurology, Neurosurgery and Psychiatry, 48, 141-144.

Bisiach E, Geminiani G, Berti A and Rusconi ML (1990) Perceptual and premotor factors of unilateral neglect. Neurology, 40, 1278-1281.

Bradshaw JL and Rogers LJ (1993) The Evolution of Lateral Asymmetries, Language, Tool Use and Intellect. Academic Press, San Diego.

Bradshaw JL, Nathan G, Nettleton NC, Pierson JM and Wilson L (1983) Head and body space to left and right. III. Vibrotactile stimulation and sensory and motor components. Perception, 12, 651-661.

Bradshaw JL, Nettleton NC, Pierson JM, Wilson LE and Nathan G (1987) Coordinates of extracorporeal space. In: Neurophysiological and Neuropsychological Aspects of Spatial Neglect (Ed M. Jeannerod), pp. 41-67. NorthHolland, Amsterdam.

Bradshaw JL, Waterfall ML, Phillips JG, Iansek R, Mattingley JB and Bradshaw JA (1993) Re-orientation of attention in Parkinson's disease: an extension to the vibrotactile modality. Neuropsychologia, 31, 51-66.

Brain WR (1941) Visual disorientation with special reference to lesions of the right cerebral hemisphere. Brain, 64, 244-271.

Caneman G, Levander M and Tegnér R (1992) A tactile maze test in unilateral spatial neglect: the influence of vision and recording technique. Journal of Neurology, 239, 273-276.

Critchley M (1966) The Parietal Lobes. Hafner Press, New York.

D'Erme P, Robertson I, Bartolomeo P, Daniele A and Gainotti G (1992) Early rightwards orienting of attention on simple reaction time performance in patients with left-sided neglect. Neuropsychologia, 30, 989-1000.

De Renzi E, Faglioni P and Scotti G (1970) Hemispheric contribution to exploration of space through the visual and tactile modality. Cortex, 6, 191-203.

De Renzi E, Gentilini M and Barbieri C (1989a) Auditory neglect. Journal of Neurology, Neurosurgery and Psychiatry, 52, 613-617.

De Renzi E, Gentilini M, Faglioni P and Barbieri C (1989b) Attentional shift towards the rightmost stimuli in patients with left visual neglect. Cortex, 25, 231-237.

Ellis AW, Flude BM and Young AW (1987) "Neglect dyslexia" and the early visual processing of letters in words and nonwords. Cognitive Neuropsychology, 4, 439-464.

Farah MJ, Wong AB, Monheit MA and Morrow LA (1989) Parietal lobe mechanisms of spatial attention: modality-specific or supramodal? Neuropsychologia, 27, 461-470.

Fujii T, Fukatsu R, Kimura I, Saso S-I and Kogure K (1991) Unilateral spatial neglect in visual and tactile modalities. Cortex, 27, 339-343.
Gainotti G, Messerli P and Tissot R (1972) Qualitative analysis of unilateral spatial neglect in relation to laterality of cerebral lesions. Journal of Neurology, Neurosurgery and Psychiatry, 35, 545-550.

Halligan PW, Marshall JC and Wade DT (1989) Visuospatial neglect; underlying factors and test sensitivity. Lancet, ii, 908-910.

Halligan PW, Marshall JC and Wade DT (1992) Contrapositioning in a case of visual neglect. Neuropsychological Rehabilitation, 2, 125-135.

Heilman KM, Bowers D, Coslett HB, Whelan $\mathrm{H}$ and Watson RT (1985a) Directional hypokinesia: prolonged reaction times for leftward movements in patients with right hemisphere lesions and neglect. Neurology, 35, 855859.

Heilman KM, Watson RT and Valenstein E (1985b) Neglect and related disorders. In: Clinical Neuropsychology, 2nd edn (Eds KM Heilman and E Valenstein), pp. 243-293. Oxford University Press, New York.

Heilman KM, Bowers D, Valenstein E and Watson RT (1987) Hemispace and hemispatial neglect. In: Neurophysiological and Neuropsychological Aspects of Spatial Neglect (Ed M. Jeannerod), pp. 115-150. North-Holland, Amsterdam.

Jeannerod M (Ed) (1987) Neurophysiological, and Neuropsychological Aspects of Spatial Neglect. North-Holland, Amsterdam.

Karnath HO, Schenkel P and Fischer B (1991) Trunk orientation as the determining factor of the "contralateral" deficit in the neglect syndrome and as the physical anchor of the internal representation of body orientation in space. Brain, 114, 1997-2014.

Kinsbourne M (1987) Mechanisms of unilateral neglect. In: Neurophysiological and Neuropsychological Aspects of Spatial Neglect (Ed M Jeannerod), pp. 69-86. NorthHolland, Amsterdam.

Kinsbourne M (1993) Orientational bias model of unilateral neglect: evidence from attentional gradients within hemispace. In: Unilateral Neglect: Clinical and Experimental Studies (Eds IH Robertson and JC Marshall), pp. 6386. Lawrence Erlbaum Associates, Hove.

Kinsella G, Olver J, Ng K, Packer S and Stark R (1993) Analysis of the syndrome of unilateral neglect. Cortex, 29, $135-140$

Làdavas E, Petronio A and Umiltà C (1990) The deployment of visual attention in the intact field of hemineglect patients. Cortex, 26, 307-317.

Mattingley JB, Bradshaw JL and Phillips JG (1992a) Impairments of movement initiation and execution in unilateral neglect: directional hypokinesia and bradykinesia. Brain, 115, 1849-1874.

Mattingley JB, Bradshaw JL and Phillips JG (1992b) Reappraising unilateral neglect. Australian Journal of Psychology, 44, 163-169.

Mattingley JB, Bradshaw JL, Bradshaw JA and Nettleton NC (1994) Residual rightward attentional bias after apparent recovery from right hemisphere damage: implications for a multicomponent model of neglect. Journal of Neurology, Neurosurgery and Psychiatry, 57, 597-604.

Meador KJ, Allen ME, Adams RJ and Loring DW (1991) Allochiria vs allesthesia: is there a misperception? $\mathrm{Ar}$ chives of Neurology, 48, 546-549.

Mesulam M-M (1985) Attention, confusional states, and 
neglect. In: Principles of Behavioral Neurology (Ed M-M Mesulam), pp. 125-167. F. A. Davis, Philadelphia.

Moscovitch M and Behrmann M (1994) Coding of spatial information in the somatosensory system: evidence from patients with neglect following parietal lobe damage. Journal of Cognitive Neuroscience, 6, 151-155.

Patterson K and Bradshaw JL (1975) Differential hemispheric mediation of nonverbal visual stimuli. Journal of Experimental Psychology: Human Perception and Performance, 1, 246-252.

Pierson JM, Bradshaw JL, Meyer TF, Howard MJ and Bradshaw JA (1991) Direction of gaze during vibrotactile choice reaction-time tasks. Neuropsychologia, 29, 925928.

Pierson-Savage JM and Bradshaw JL (1987) Mapping of extracorporeal space by vibrotactile reaction times: a far left-side disadvantage. Perception, 16, 283-290.

Pierson-Savage JM, Bradshaw JL, Bradshaw JA and Nettleton NC (1988) Vibrotactile reaction times in unilateral neglect. Brain, 111, 1531-1545.

Posner MI, Walker JA, Friedrich FJ and Rafal RD (1984) Effects of parietal injury on covert orienting of attention. Journal of Neuroscience, 4, 1863-1874.

Posner MI, Walker JA, Friedrich FA and Rafal RD (1987) How do the parietal lobes direct covert attention? Neuropsychologia, 25, 135-145.

Robertson IH and Cashman E (1991) Auditory feedback for walking difficulties in a case of unilateral neglect. Neuropsychological Rehabilitation, 1, 170-175.

Robertson IH and Marshall JC (Eds) (1993) Unilateral
Neglect: Clinical and Experimental Studies. Lawrence Erlbaum Associates, Hove.

Tegnér R and Levander M (1991) Through a looking glass. A new technique to demonstrate directional hypokinesia in unilateral neglect. Brain, 114, 1943-1951.

Vallar G, Rusconi ML, Geminiani G, Berti A and Cappa SF (1991) Visual and nonvisual neglect after unilateral brain lesions: modulation by visual input. International Journal of Neuroscience, 61, 229-239.

Villardita C (1987) Tactile exploration of space and visual neglect in brain-damaged patients. Journal of Neurology, 234, 292-297.

Weintraub S and Mesulam M-M (1987) Right cerebral dominance in spatial attention. Archives of Neurology, 44, 621-625.

Weiskrantz L and Zhang D (1987) Residual tactile sensitivity with self-directed stimulation in hemianaesthesia. Journal of Neurology, Neurosurgery and Psychiatry, 50, 632634.

Wilson B, Cockburn J and Halligan PW (1987) Behavioural Inattention Test. Thames Valley Test Company, Titchfield, Hants.

Wood RG, Bradshaw JL, Pierson-Savage JM, Nettleton NC and Bradshaw JA (1988) Vibrotactile stimulator for use with patients suffering from hemineglect. Medical \& Biological Engineering \& Computing, 26, 207-212.

(Received 4 January 1994; accepted as revised 16 June 1994) 


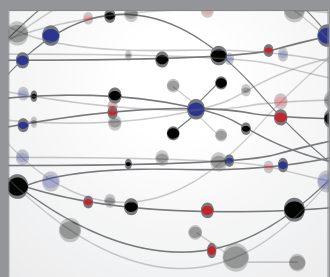

The Scientific World Journal
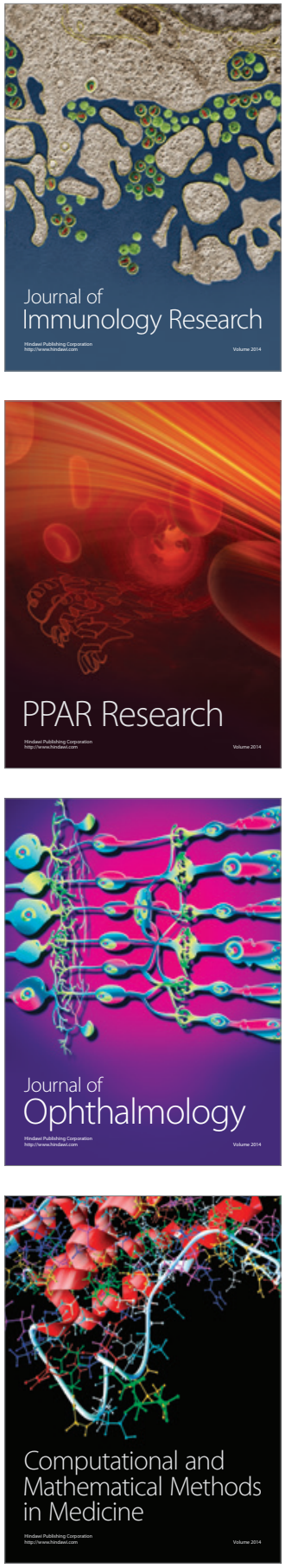

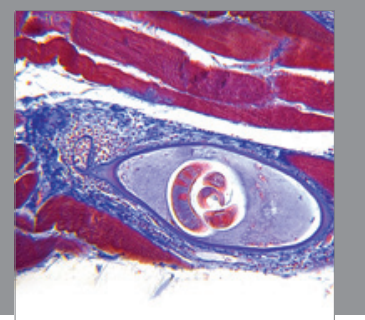

Gastroenterology

Research and Practice
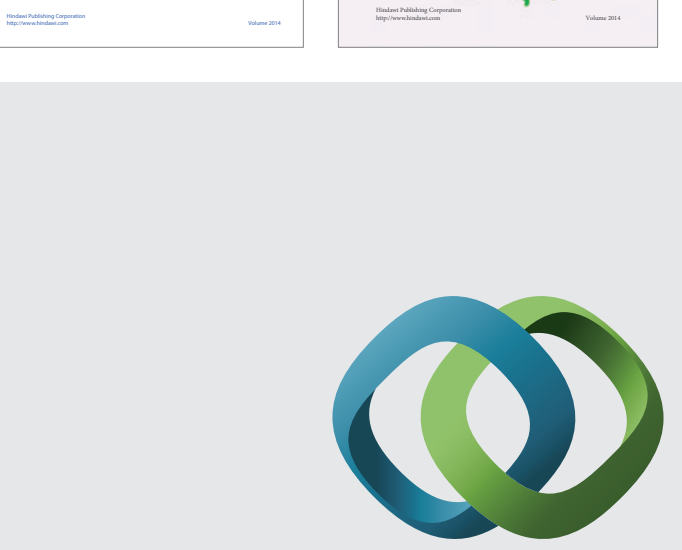

\section{Hindawi}

Submit your manuscripts at

http://www.hindawi.com
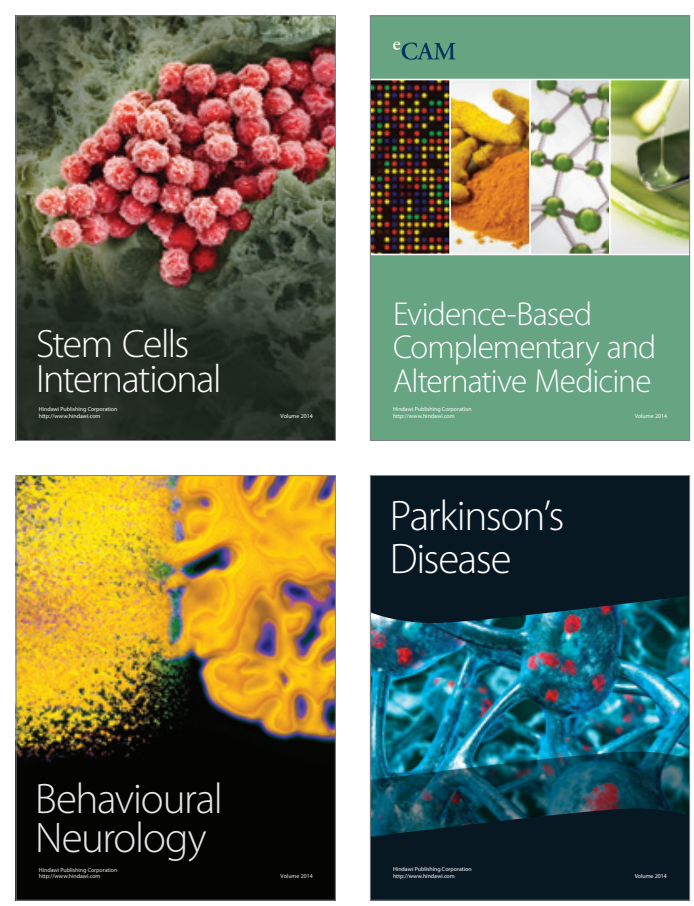

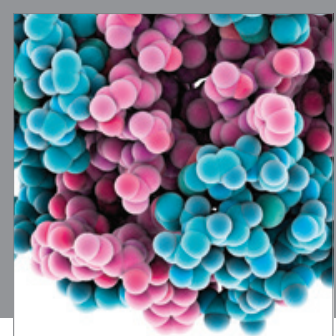

Journal of
Diabetes Research

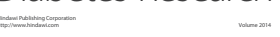

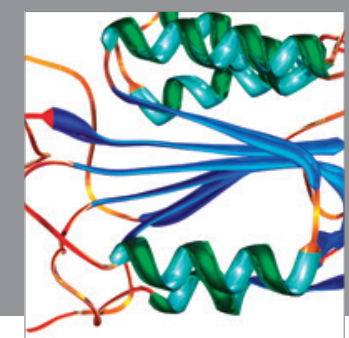

Disease Markers
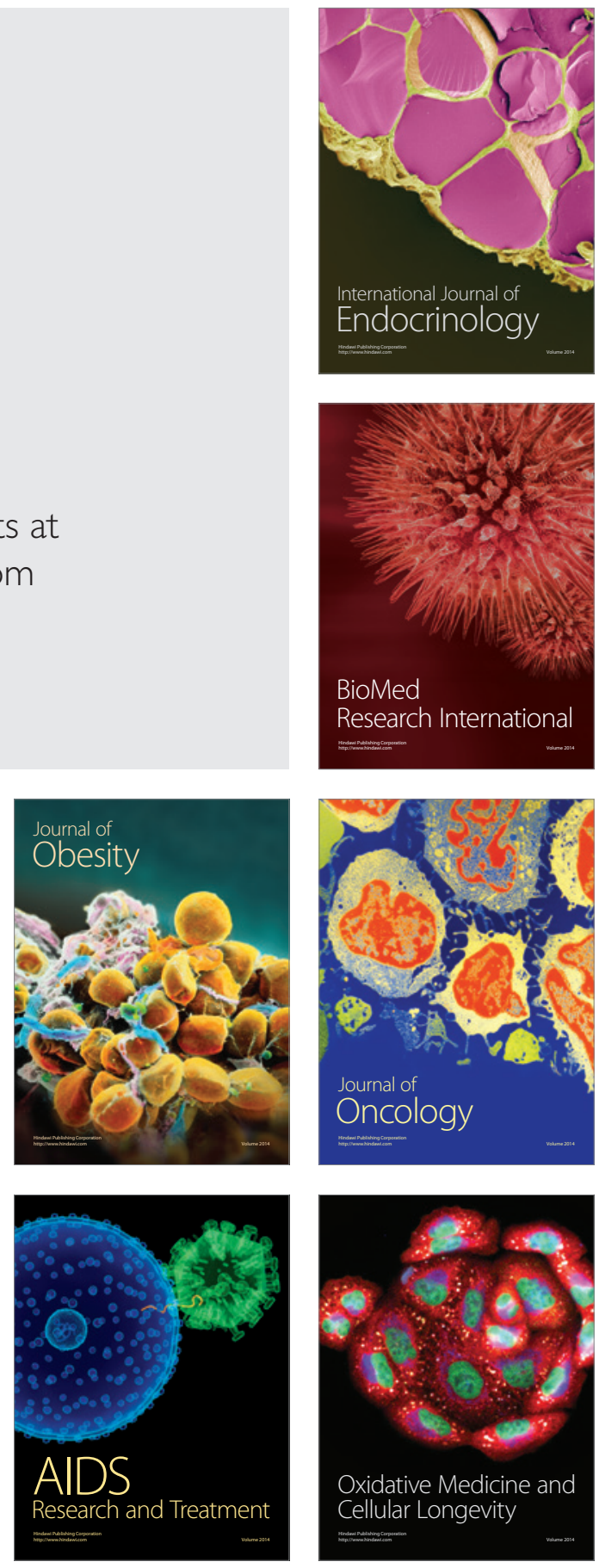